\title{
Huanglian Jiedu Decoction ameliorates DSS-induced colitis in mice via the JAK2/STAT3 signalling pathway
}

\author{
Zhuo Lu ${ }^{1 \dagger}$, Wanna Xiong ${ }^{2 \dagger}$, Simeng Xiao ${ }^{1}$, Yilong Lin ${ }^{1}$, Kai Yu$^{3}$, Guihua Yue ${ }^{2}$, Qiaoming Liu' ${ }^{1}$, Fang Li ${ }^{1}$ \\ and Jianqin Liang ${ }^{1 *}$
}

\begin{abstract}
Background: Ulcerative colitis (UC) is an intestinal disease which was characterized by intestinal inflammation, mucosal injury and fibrosis. In this paper, the effect of Huanglian Jiedu Decoction (HJD), a well-known traditional Chinese medicine with significant anti-inflammatory effect, on dextran sulphate sodium (DSS)-induced UC in mice and inhibition of JAK2/STAT3 pathway were investigated.
\end{abstract}

Methods: BALB/C mice were randomly divided into 6 groups: HJD group (high, medium and low dose), USAN group, UC group, and control group. UC in mice were induced through free access to 3\% DSS solution. After being treated with HJD for 8 days, all animals were sacrifice. Pathological examination of colonic specimen was performed by H\&E staining. Cytokines (TNF-a, IL-6, and IL-1 $\beta$ ) in colon were assayed by ELISA and immunofluorescence, MPO in colon and ATT in serum were detected by ELISA. Moreover, mice in HJD group and UC group were treated with AG490 to inhibit the expression of JAK2 protein, then the expression of JAK2 and STAT3 protein in colon was determined by western blotting and immunofluorescence staining. Furthermore, KI67 in colon was examined by immunohistochemistry, and apoptosis was detected by TUNEL staining, and collagen deposition was assayed by Masson staining after JAK2/STAT3 pathway in UC mice was inhibited by HJD.

Results: After mice being treated with HJD, the symptoms (weight loss and haematochezia) of UC were alleviated, and the contents of inflammatory cytokines (TNF-a, IL- 6 and IL-1 $\beta$ ) and MPO in colon were significantly decreased. The expression of JAK2 and STAT3 protein was reduced after administration with HJD. After JAK2/STAT3 pathway being inhibited with HJD, the cell apoptosis, collagen deposition and immunoreactivity of macrophage in colon were significantly reduced, but the expression of Ki67 was markedly enhanced in both UC group and HJD group compare with control group.

Conclusions: HJD treatment can alleviate intestinal mucosal damage and has the protective effect on UC by downregulating JAK2 and STAT3 expression to reduce inflammation via JAK2/STAT3 pathway.

Keywords: Ulcerative colitis, Huanglian Jiedu Decoction, JAK2/STAT3, Dextran sulphate sodium

*Correspondence: 000298@gxtcmu.edu.cn

†Zhuo Lu and Wanna Xiong contributed equally to this work

${ }^{1}$ Guangxi University of Chinese Medicine, Nanning 530001, China

Full list of author information is available at the end of the article

\section{Background}

Ulcerative colitis (UC) is an intestinal disease that is characterized by intestinal inflammation, mucosal injury, and fibrosis. It will be prone to develop to colorectal cancer with a probability about $5 \%-10 \%$ in the development of "inflammation-atypical hyperplasia-cancer" $[1,2]$. 5-ASA, immunosuppressive agents, monoclonal

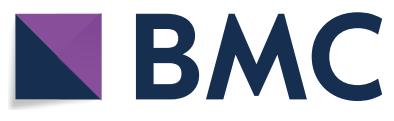

(c) The Author(s) 2020. This article is licensed under a Creative Commons Attribution 4.0 International License, which permits use, sharing, adaptation, distribution and reproduction in any medium or format, as long as you give appropriate credit to the original author(s) and the source, provide a link to the Creative Commons licence, and indicate if changes were made. The images or other third party material in this article are included in the article's Creative Commons licence, unless indicated otherwise in a credit line to the material. If material is not included in the article's Creative Commons licence and your intended use is not permitted by statutory regulation or exceeds the permitted use, you will need to obtain permission directly from the copyright holder. To view a copy of this licence, visit http://creativeco mmons.org/licenses/by/4.0/. The Creative Commons Public Domain Dedication waiver (http://creativecommons.org/publicdomain/ zero/1.0/) applies to the data made available in this article, unless otherwise stated in a credit line to the data. 
antibodies (Infliximab), and glucocorticoids are the effective therapeutic drugs for UC. However, after a long-term use of these drugs, it is easy to bring toxicity, allergy, upper gastrointestinal bleeding, and other side effects [3].

The etiology and pathogenesis of UC remains unknown. It is commonly believed that the abnormalities of intestinal immunity and intestinal flora balance in the early stage of UC were the pathogenesis $[4,5]$. Intestinal mucosal damage usually leads to a burst of cytokines (IL6 , IL-1 $\beta$, TNF- $\alpha$, etc.). The excessive expression of IL-6 can deepen the infiltration of macrophages and neutrophils, and also promote cell apoptosis and autophagy via JAK2/STAT3 pathway, which results in much severer damage [6]. The intestinal impairment was associated with the abnormally expression of STAT3 and JAK2 protein $[7,8]$.

HJD consists of four herbal materials, including Rhizoma coptidis (HL), Cortex phellodendri chinensis (HB), Radix scutellariae (HQ) and Fructus gardeniae (ZZ)), which are widely used in the treatment of sepsis, arthritis, autoimmune diseases, and intestinal diseases in clinical practice [9-11]. Its anti-inflammatory effects could be related to the action on multiple protein targets [12]. However, the therapeutic effect of HJD on UC and its mechanism are still unclear. In this paper, the effect of HJD on UC and its regulation on the JAK2/STAT3 pathway were investigated, the effects of HJD on the apoptosis and proliferation which were regulated by JAK2/ STAT3 pathway in colon were detected.

\section{Methods}

\section{Chemical reagents}

Dextran sulphate sodium (DSS; molecular weight 36,000-50,000 Da, HPLC $\geq 97 \%$ purity) was purchased from MP Biomedicals Inc. (Santa Ana, CA, USA). Dimethyl sulphoxide (DMSO) and Tyrphostin AG490 were purchased from Sigma-Aldrich (St. Louis, Missouri, USA). OB reagent was purchased from Zhuhai Besso Biotechnology Company (Zhuhai, China). Mesalazine (USAN; L/N: 16J05289L) was purchased from Losan Pharma GmbH (Frankfurt, Germany).

\section{Preparation of HJD}

HL, HB, HQ and ZZ were purchased from Nanning Wanyaotang Pharmaceutical Co., Ltd. (Nanning, China) and authenticated by Professor Songji Wei (College of Pharmacy, Guangxi University of Chinese Medicine, China). The voucher specimens, deposited at the Guangxi University of Chinese Medicine, were HL-20170401, HQ-2017-0402, HB-2017-0403, and ZZ-2017-0104 for $\mathrm{HL}, \mathrm{HQ}, \mathrm{HB}$, and $\mathrm{ZZ}$, respectively.

Briefly, $300 \mathrm{~g}$ of HL, $200 \mathrm{~g}$ of HB, $200 \mathrm{~g}$ of HQ, and $300 \mathrm{~g}$ of $\mathrm{ZZ}$ were extracted twice, for $2 \mathrm{~h}$ each time, by refluxing in water according to the weight ratio of $15: 1$ of water to herbal. Then, the aqueous solution was combined, then filtered and concentrated in a rotary evaporator to a fluid extract with a relative density of about 1.05-1.20 (measured at $50-60{ }^{\circ} \mathrm{C}$ ), and then was stored in a refrigerator at $4{ }^{\circ} \mathrm{C}$. The content of berberine hydrochloride, a kind of active compound, in HJD extract was $20.39 \mathrm{mg} / \mathrm{g}$, which was analyzed by high-performance liquid chromatography (Alliance 2695, Waters, USA) on a Inertsil ODS-2 C18 analytical column $(4.6 \mathrm{~mm} \times 250 \mathrm{~mm}, 5 \mu \mathrm{m})$ with elution by acetonitrile- $0.05 \mathrm{moL} / \mathrm{L}$ phosphoric acid aqueous solution (50:50), a flow rate at $1.0 \mathrm{~mL} / \mathrm{min}$ and the detection wavelength of $345 \mathrm{~nm}$. The column temperature was $30^{\circ} \mathrm{C}$, and the injection volume was $10 \mu \mathrm{L}$.

\section{Animal treatment}

Four-week-old male BALB/c mice (18 \pm 2 g) were purchased from Hunan SJA Laboratory Animal Co., Ltd. (Hunan, China) with license No. SCXK (Hunan) 20160002. All mice were fed at $25{ }^{\circ} \mathrm{C} \pm 2{ }^{\circ} \mathrm{C}$ and $50 \% \pm 5 \%$ relative humidity $(\mathrm{RH})$ with a 12 -h light/dark cycle with free access to standard water and food.

In the pharmacodynamics experiment, mice $(n=60)$ were randomly divided into 6 groups, namely the control group (Control; $10 \mathrm{~mL} / \mathrm{kg}, 0.9 \%$ saline), UC group (UC; 3\% DSS), high-HJD dose group (H-HJD; $24.66 \mathrm{~g} /$ $\mathrm{kg}$, weight ratio between crude drug and mice), mediumHJD dose group (M-HJD; $12.33 \mathrm{~g} / \mathrm{kg}$ ), low-HJD dose group (L-HJD; $6.17 \mathrm{~g} / \mathrm{kg}$ ), and USAN group (USAN; $0.3 \mathrm{~g} / \mathrm{kg}$ ). In the JAK2/STAT3 pathway blocking experiments, mice $(n=60)$ were randomly divided into a control group (Control; $10 \mathrm{~mL} / \mathrm{kg}, 0.9 \%$ saline), UC group (UC; 3\% DSS), AG490 group (AG490; $0.01 \mathrm{~g} / \mathrm{kg}$ ), HJD group (HJD; $24.66 \mathrm{~g} / \mathrm{kg}$ ), and interference group (AG490+ HJD; 0.01 g/kg AG490 + 24.66 g/kg HJD). Each group consisted of 10 mice.

After 3 days of acclimation, mice free drank 3\% DSS solution $(\mathrm{w} / \mathrm{w})$ to induce UC except the mice in control group. For the HJD treatment group and USAN group, HJD and USAN were administrated by gavage once a day at a volume of $10 \mathrm{~mL} / \mathrm{kg}$. The AG490 group was subjected to intraperitoneal injection. The UC group and control group were subjected to gastric lavage with normal saline. All mice were treated for 8 days.

Then, mice were euthanized by cervical dislocation on day 8. Serum was obtained after centrifugation at $3000 \mathrm{rpm}$ for $10 \mathrm{~min}$ at $4{ }^{\circ} \mathrm{C}$ and was stored at $-20{ }^{\circ} \mathrm{C}$. Colon tissues from the anus to the ileum were collected and cut in half. One half was fixed in 4\% neutral-buffered formalin for histopathological and immunohistochemistry (IHC) analysis, the other half was frozen in liquid nitrogen and stored at $-80^{\circ} \mathrm{C}$ for western blotting assays. 


\section{Colon macroscopic damage index (CMDI)}

The congestion, oedema, atrophy, and ulcer of the colon were surveyed after mice had been dissected, and CMDI was evaluated according to the previously published criteria [13]: 0: no obvious congestion, oedema or ulcer; 1: local congestion, oedema but no ulcer; 2: scattered ulcer spots; 3: linear continuous distribution of ulcer points, inflammatory oedema, hyperaemia $<1 \mathrm{~cm}$; 4 : formation of ulcer lesions, inflammatory oedema, hyperaemia range $>1 \mathrm{~cm}$.

\section{Disease activity index (DAI)}

All mice were weighed, the faecal characteristics were observed, and faecal occult blood tests were performed every day. The DAI was evaluated according to a previously published criteria [14]: 0: 0\% weight loss, normal stool and negative occult blood test; 1 : $1-5 \%$ weight loss, a normal stool, and negative occult blood test; 2 : 6-9\% weight loss, a soft stool, and positive occult blood test; 3: 10-15\% weight loss, a soft stool, and positive occult blood test; $4:>15 \%$ weight loss, diarrhoea, and visible bleeding.

\section{Haematoxylin and eosin (H\&E) staining}

Colon tissues were fixed with $4 \%$ neutral-buffered formalin for $24 \mathrm{~h}$. After that the colon was eluted with gradient ethanol and then immersed in xylene for $1 \mathrm{~h}$. The paraffin-embedded colon samples were sliced into $4-\mu \mathrm{m}$-thick sections, stained with haematoxylin and eosin, imaged under a light microscope for routine histopathological examination, and then graded according to the scoring criteria [15]: 0: no inflammatory cell infiltration, no goblet cell damage; 1 : inflammatory cell immersion and local loss of goblet cells in the mucosal layer; 2: inflammatory infiltration in the mucosa, spreading to the submucosa, and loss of multiple sites of goblet cells; 3: inflammation continuously distributed in the mucosa and submucosa, with the loss of a large number of goblet cells; 4: full-thickness inflammatory cells seriously infiltrated and goblet cells completely damaged.

\section{Enzyme-linked immunosorbent assay (ELISA)}

The contents of TNF- $\alpha$, IL- 6, IL- $1 \beta$ and MPO in the colonic tissue, and $\alpha-1$ antitrypsin (ATT) in serum (Nanjing Jiancheng Bioengineering Institute, China) were determined by ELISA according to the manufacturer's instructions.

\section{Western blotting (WB)}

The colon tissue was added to radioimmunoprecipitation assay lysate (containing a protease inhibitor), and the supernatant was drained after homogenization. The protein concentration was determined using the bicinchoninic acid method after gradient dilution. The proteins were separated by SDS-PAGE and transferred to a polyvinylidene difluoride membrane (Millipore, USA). Membranes was sealed at room temperature for $1 \mathrm{~h}$ and then incubated with primary antibodies against JAK2 (D2E12, Cell Signaling Technology, CST Inc., Beverly, MA, USA), STAT3 (124H6, CST Inc.), and phosphoSTAT3 (Ser727, CST Inc.) overnight at $4{ }^{\circ} \mathrm{C}$. Then the membranes were washed 3 times and incubated with a horseradish peroxidase (HRP)-conjugated secondary antibody for $1 \mathrm{~h}$ at room temperature (RT). The bands were semi-quantitated with Image J (National Institutes of Health, USA) software.

\section{Immunohistochemistry (IHC)}

The 4- $\mu \mathrm{m}$-thick slices were mounted on glass slides and baked for $1.5 \mathrm{~h}$ at $50{ }^{\circ} \mathrm{C}$, after which they were deparaffinized and the endogenous peroxidase activity quenched. The primary antibody Ki67 (ZA-502, 1:1000 dilution) was incubated on the slides for $12 \mathrm{~h}$ at $4{ }^{\circ} \mathrm{C}$. After rinsing three times with phosphate-buffered saline solution containing Tween, the horseradish peroxidase-conjugated secondary antibody (ZSGB-BIO, PV-9001) was incubated for $20 \mathrm{~min}$ at $\mathrm{RT}$ and then visualized after incubation with 3, 3-diaminobenzidine for $10 \mathrm{~min}$ at RT, after which the binding sites were sealed with neutral resin. Terminal deoxynucleotidyl transferase dUTP nick end labelling (TUNEL) analysis was performed on the colon tissue using an In-Situ Cell Death Detection Kit (Roche, 1:100 dilution). Images were obtained at $20 \times$ the original magnification. The average optical density (AOD) was measured by Image J software.

\section{Immunofluorescence staining}

Colon tissue was sealed with wax and sectioned into $4 \mu \mathrm{m}$ thick slices. Sectioned samples were deparaffinized in xylene, rehydrated with gradient alcohol, and subjected to antigen retrieval. Colon sections were washed with PBS five times for $3 \mathrm{~min}$ each. Then, $0.5 \%$ (wt/vol) Triton X-100 and blocking serum were added successively and incubated for $10 \mathrm{~min}$ and $1.5 \mathrm{~h}$, respectively. The tissue was incubated in primary antibody, rabbit TNF- $\alpha$ (1:200, AF7014, Affinity Biosciences Inc., China), rabbit IL1- $\beta$ (1:200, AF5103, Affinity Biosciences Inc., China), rabbit IL-6 (1:200, DF6087, Affinity Biosciences Inc., China), rabbit JAK2 (1:200, AF6022, Affinity Biosciences Inc., China), rabbit STAT3 (1:200, AF6294, Affinity Biosciences Inc., China), rabbit F4/80(1:200, DF7762, Affinity Biosciences Inc., China), at $4{ }^{\circ} \mathrm{C}$ overnight. After being washed four times with PBS, the sections were incubated with the secondary antibody (ZF-0516, Alexa Fluor ${ }^{\circledR} 594$, 
ZSGB-Bio Inc., China) for $2 \mathrm{~h}$ at $\mathrm{RT}$ and protected from light. Images were obtained at $20 \times$ the original magnification. The relative area immunoreactivity was calculated with Image J software.

\section{Masson staining}

After dewaxing, sectioned samples was stained with hematoxylin for $30 \mathrm{~s}$, hydrochloric acid to induce differentiation into blue, Masson compound staining solution for $2 \mathrm{~min}, 1 \%$ phosphomolybdate and bright green staining solution for $5 \mathrm{~min}$, respectively. The sections were washed 3 times with $0.2 \%$ acetic acid after each staining, then dehydrated with anhydrous alcohol, cleaned with xylene until transparent, and sealed tightly with neutral glue. Images were obtained at $20 \times$ and $40 \times$ the original magnification. The collagen fibbers in tunica mucosa and submucosa was calculated with Image J software.

\section{Statistical analysis}

Statistical data are expressed as mean \pm standard deviation (SD), and a one-way analysis of variance (ANOVA) followed by Dunnett's t-test was used for comparisons between groups. The data were analysed with trial version SPSS (SPSS Inc., Chicago, IL, USA). $P<0.05$ was regarded as statistically significant.

\section{Results}

\section{HJD treatment attenuated the symptom in DSS-induced UC mice}

Contrary to the stable increase in body weight, soft stool, and negative occult blood test in control group, there were significant changes in UC group on the 5th day. The weight loss of the mice in HJD group and USAN group was less significant than in UC group (Fig. 1a). Diarrhoea and haematochezia were found in the groups treated with 3\% DSS (UC group, HJD group and USAN group) on days 3 to 8 . The different was that diarrhoea and haematochezia in HJD group and USAN group was less severe than that in UC group. Therefore, the DAI was significantly increased in UC group compared with control group. Meanwhile, a lower DAI was demonstrated in HID group and USAN group (Fig. 1b). Moreover, the CMDI and histology scores in UC group were significantly higher than that in other groups (Fig. 1c, d).

As shown in Fig. 1e, there was significant shortening of the colon length in UC group, with obvious congestion, oedema, and atrophy compared with control group. These symptoms were much less severe in HJD group and USAN group. These results indicated that HJD significantly ameliorated the symptoms of UC in mice, including weight loss, diarrhoea, haematochezia, and oedema. As shown by H\&E staining (Fig. 1f), it exhibited an intact epithelial layer and crypt-villus structures in control group. However, the other groups exhibited histopathological injuries, including inflammatory cell infiltration and a paucity of intact crypt-villus structures. Interestingly, HJD and USAN significantly alleviated the intestinal damage through enhancing the intestinal mucosa integrity, and attenuating the inflammatory infiltration.

\section{HJD treatment reduced the production of cytokines (TNF- $\alpha$, IL-6, and IL-1 $\beta$ ) and MPO in colon tissues, and enhanced ATT level in serum}

The results (Fig. $2 \mathrm{a}-\mathrm{c}$ ) showed that the contents of TNF- $\alpha$, IL-6, and IL-1 $\beta$ in UC group were significantly increased compared with control group $(P<0.01)$. After mice being treated with USAN and HJD, the concentrations of TNF- $\alpha$, IL- 6 , and IL- $1 \beta$ were significantly decreased compared with UC group $(P<0.05)$. In addition, we analyzed these proteins by immunofluorescence analysis, similar results were obtained (Fig. 2f-i). Moreover, as shown in Fig. 2d-e, HJD treatment MPO were significantly decreased increased the ATT level $(P<0.01)$.

\section{HJD inhibited the protein expression of JAK2 and STAT3}

AG490, a type of JAK2 inhibitor, was used to block the JAK2/STAT3 pathway. As shown in Fig. 3, the mice treated with AG490 (AG490 group and AG490 + HJD group), and HJD group significantly attenuated weight loss, diarrhoea, haematochezia, and oedema, with a lower CMDI and histology scores compared with UC group $(P<0.05)$. They exhibited a therapeutic effect to $\mathrm{UC}$ in mice.

WB assay (Fig. 4a, b) showed that the expression of JAK2 and STAT3 in UC group were significantly increased compared with control group $(P<0.01)$, while their expression in AG490 group and HJD group were significantly downregulated $(P<0.01)$. Similar results obtained by immunofluorescence analysis of JAK2 and STAT3 protein (Fig. 4c-e). This indicated that HJD exhibited an inhibition of JAK2/STAT3 pathway on UC in mice.

\section{Changes of K167, apoptosis, collagen deposition and immunoreactivity of macrophage in colon after inhibition of JAK2/STAT3 pathway with HJD} Normal physiological proliferation, apoptosis, differentiation, migration, and tight junctions of intestinal epithelial cells, which are regulated through the JAK2/STAT3 pathway, are essential for maintaining the integrity of the intestinal mucosa. In this paper, IHC and TUNEL were applied to detect Ki67 expression and apoptosis in colonic epithelial cells, respectively. The results (Fig. 5ac) showed an increase in Ki67 and a decrease in apoptosis in both UC group and HJD group compared with control group. To characterize the infiltrated macrophage 


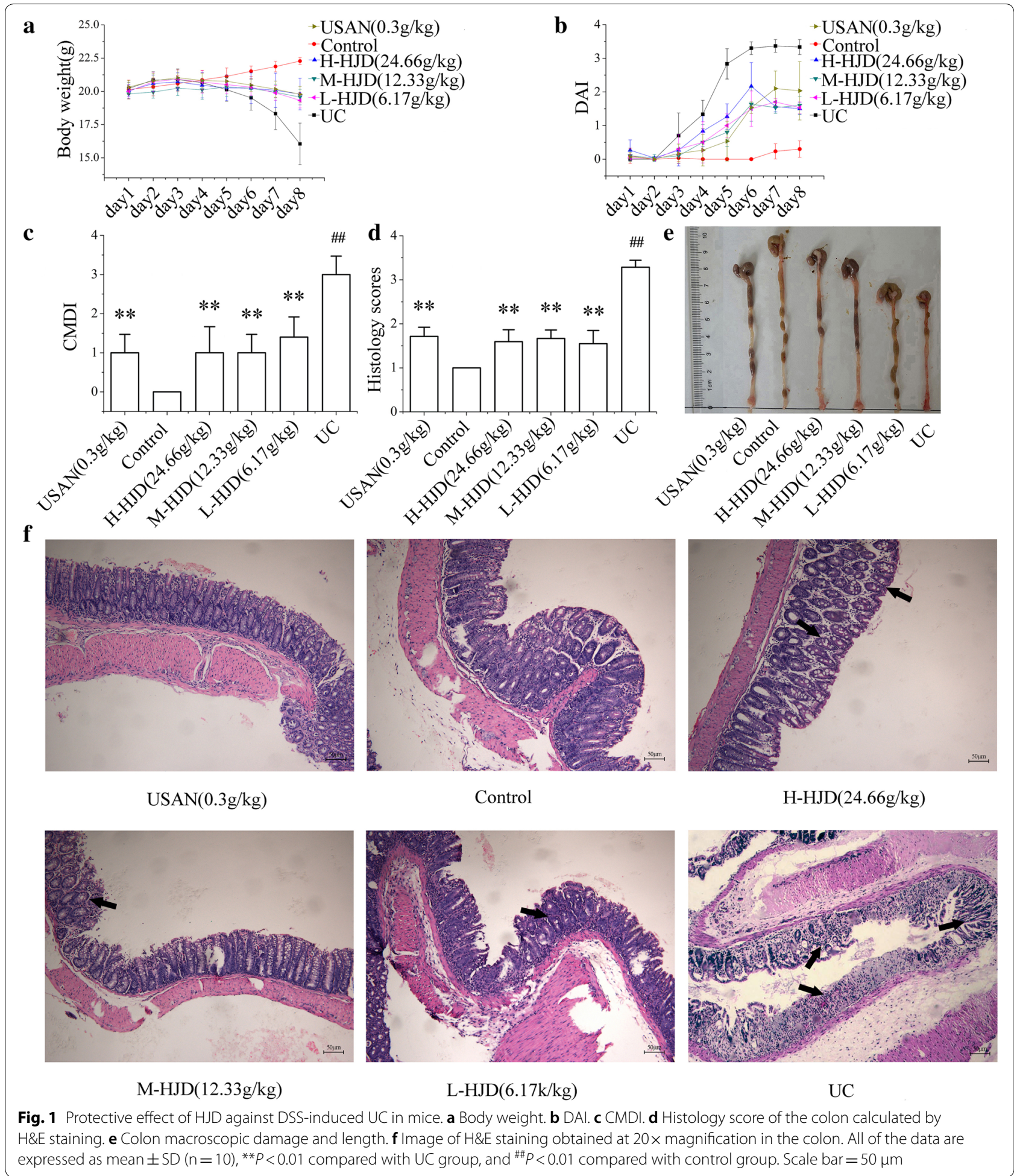

population in the colon, immunofluorescence staining was performed for macrophages phenotypic markers F4/80. The results showed an increase of F4/80 macrophages in UC group compared with control group
$(P<0.01)$, and a decrease of $F 4 / 80$ macrophages was observed in HJD group (Fig. 5a, d).

Collagen fibrosis is a pathological manifestation of many diseases. The abnormal accumulation and degradation 


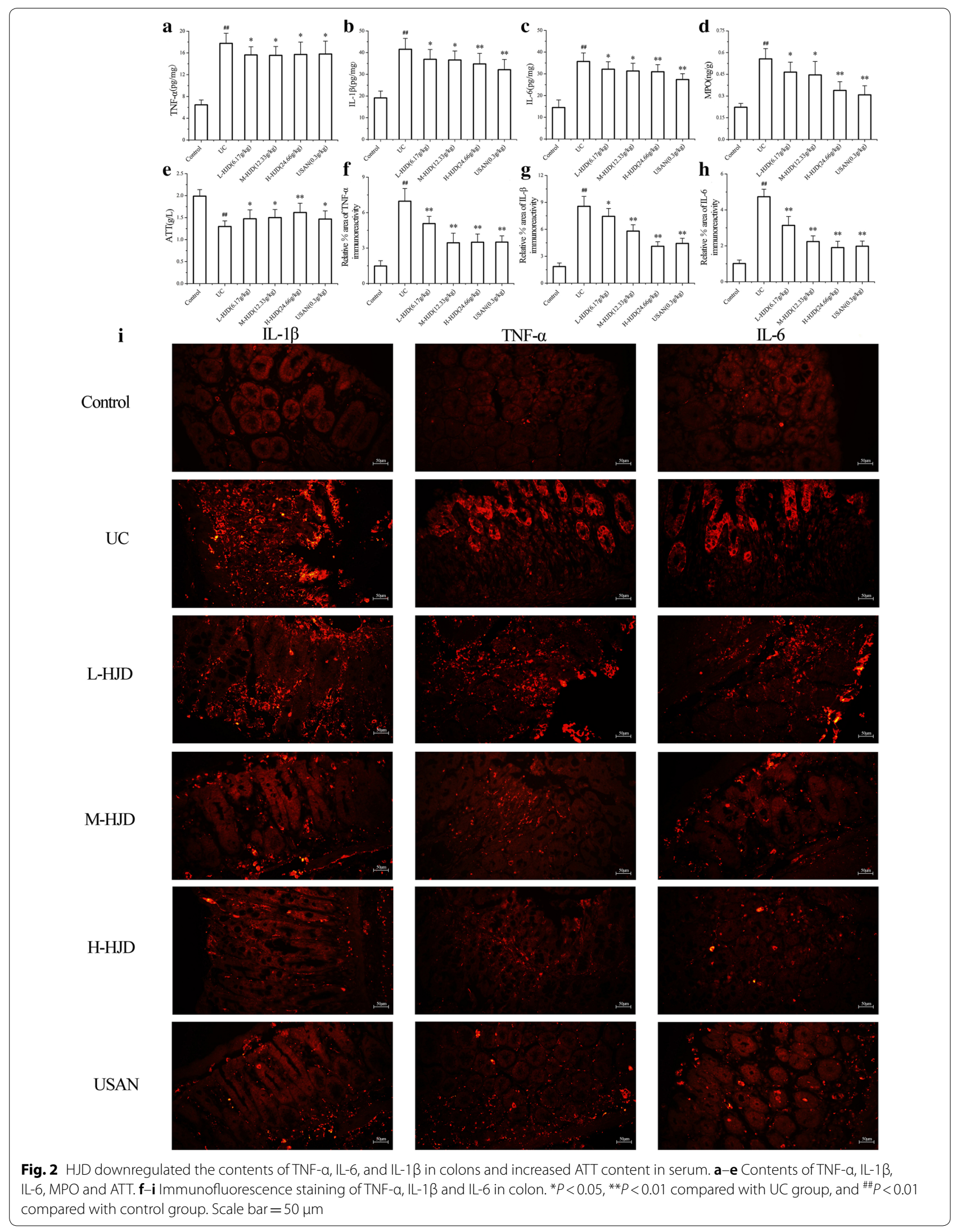




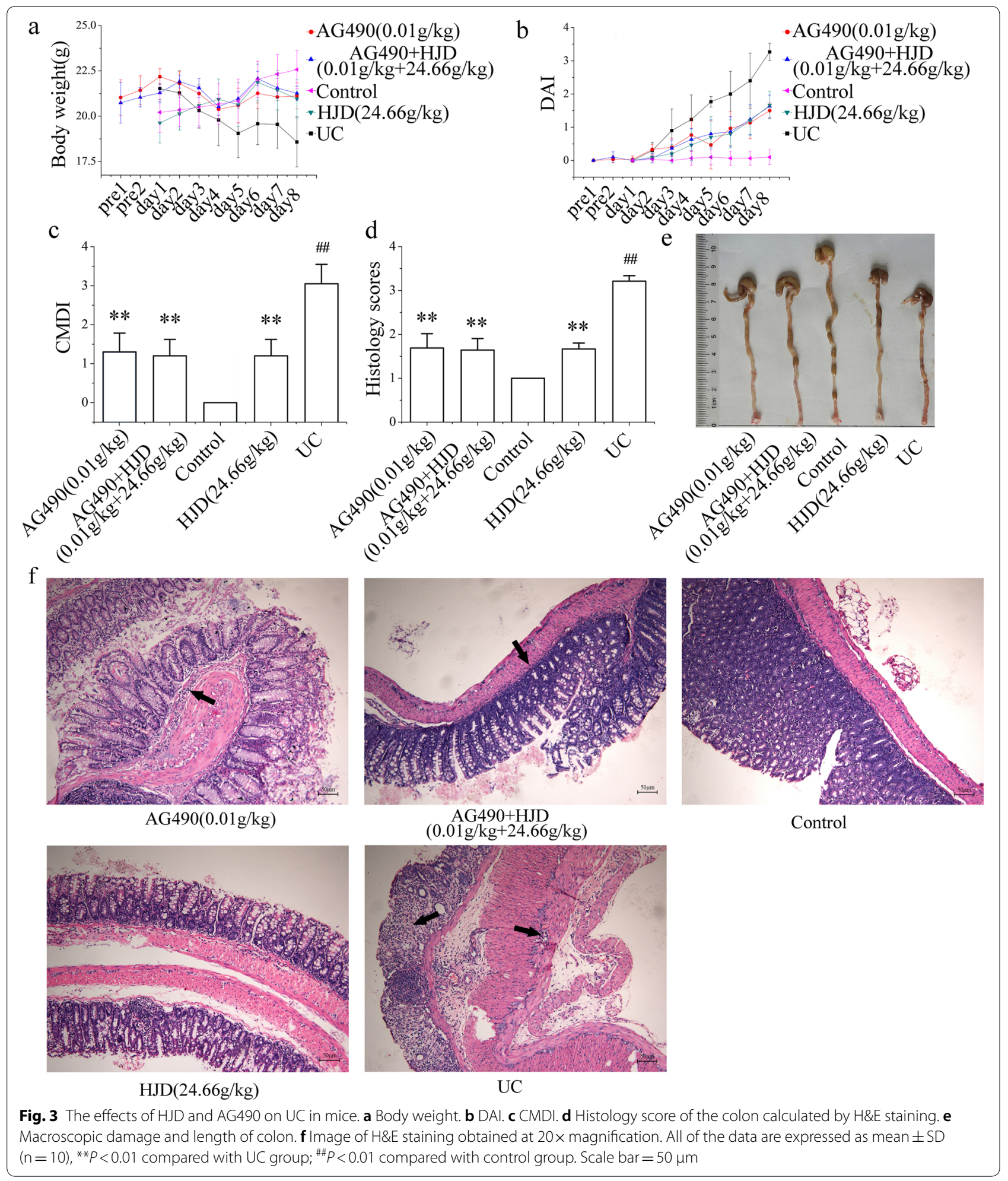

of collagen are the basis of fibrosis. It would bring about intestinal fibrosis in UC patient. Since apoptosis easily leads to the disruption of intestinal mucosal integrity and barrier function, which leads to inflammation and intestinal fibrosis, the expression of collagen in colonic mucosa of UC mice was performed by Masson staining. As shown in 


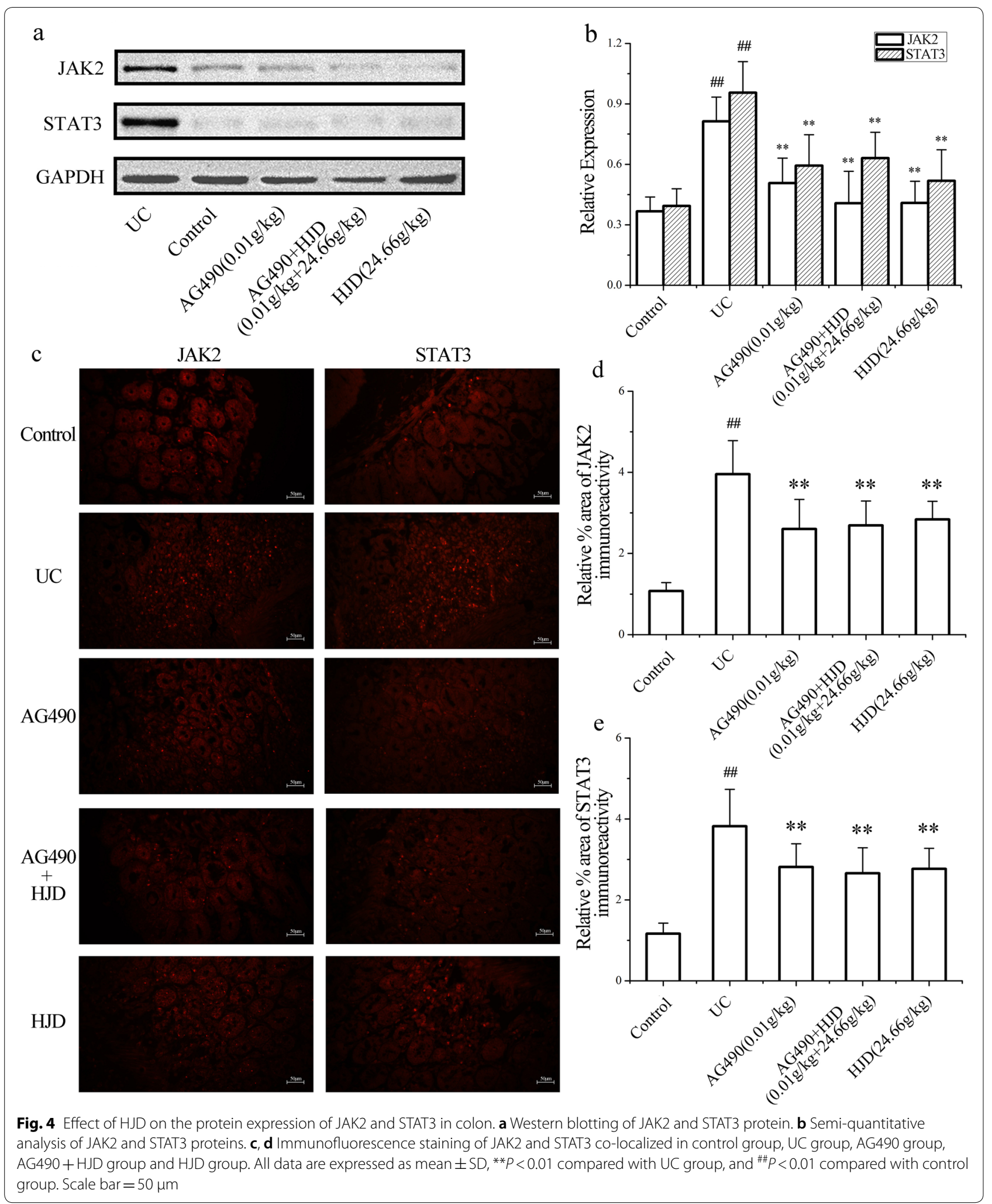




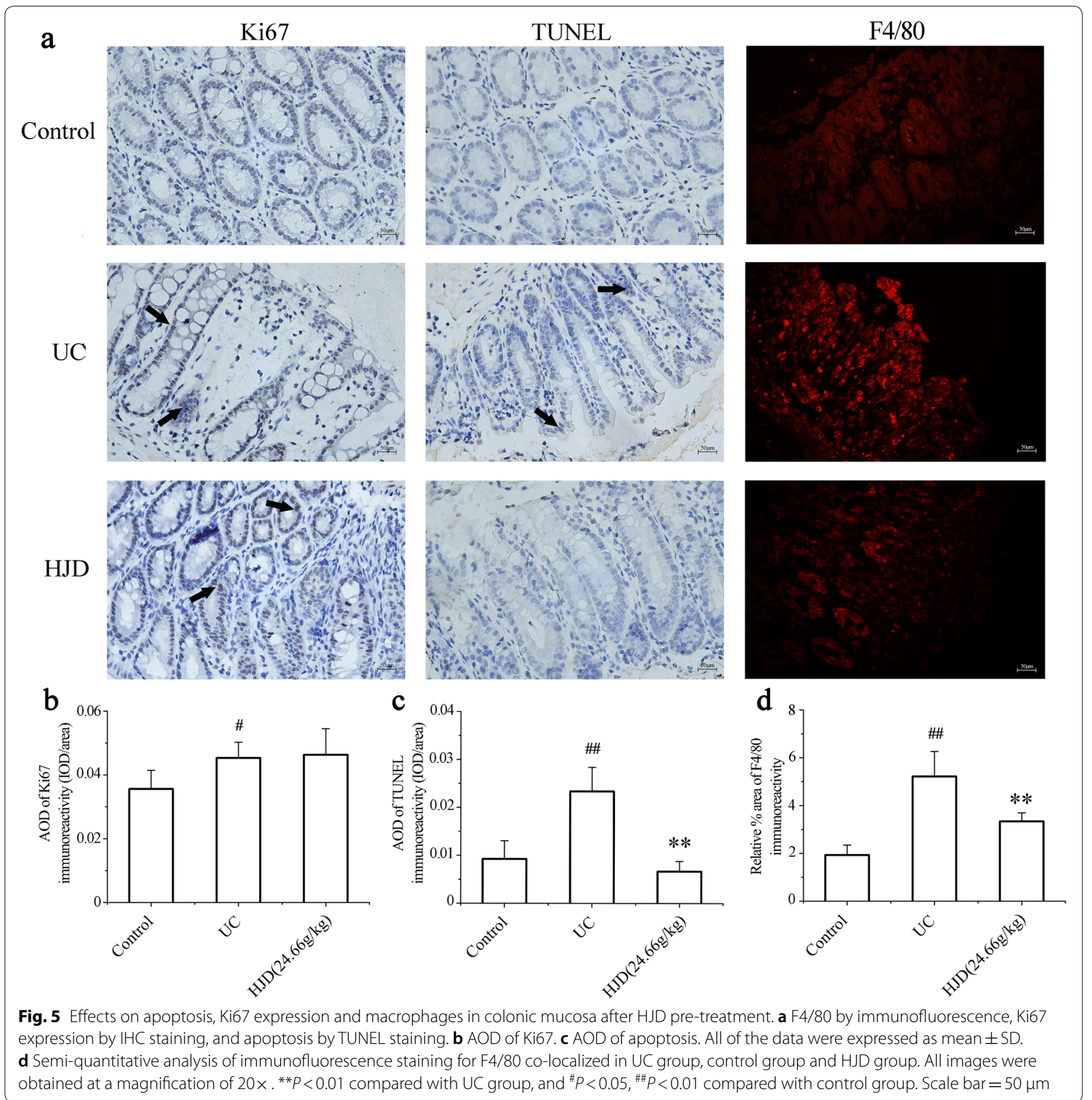

Fig. 6, the expression of collagen proteins in UC group was significantly increased $(P<0.01)$ compared with control group, while in HJD group it was significantly decreased $(P<0.01)$ compared with UC group. These results suggested that HJD treatment could reduce the risk of intestinal fibrosis in UC.

\section{Discussion}

UC is characterized by intestinal inflammation and mucosal damage. The damage of intestinal mucosa is crucial to the occurrence and development of UC. The DSS-induced colitis mouse model was widely used to study the pathogenesis of UC and screen therapeutic 


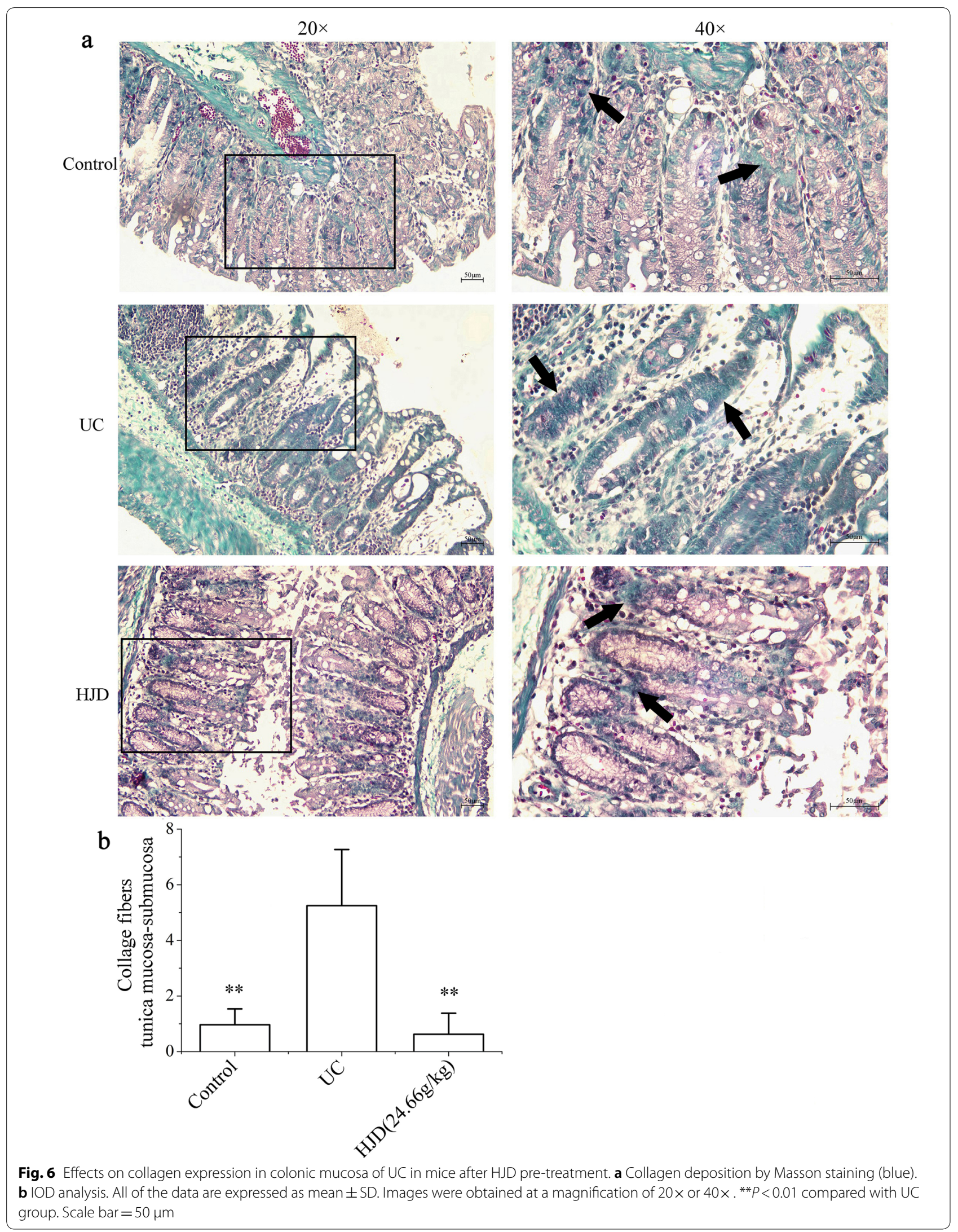


drugs [16]. In the acute stage of $\mathrm{UC}$, the release of inflammatory factors (TNF- $\alpha$, IL-1 $\beta$, and IL-6) will aggravate the accumulation and infiltration of inflammatory cells in colonic tissue $[17,18]$. The excessive inflammation will further destroy the integrity of the intestinal barrier, which would lead to the formation of intestinal oedema and ulceration [7]. Inhibiting the expression of inflammatory cytokines will exhibit a therapeutic effect on UC [19-21]. Our results demonstrated that HJD treatment markedly alleviated colonic mucosal injury in UC mice, reduced the level of inflammatory cytokines (IL-1 $\beta$, IL-6, and TNF-a) and MPO in colon, and increased the content of ATT.

JAK2/STAT3 pathway is an important and classical inflammatory pathway. JAK2 and STAT3 proteins are the key regulators of inducible expression of many genes that are involved in inflammatory responses in the intestinal tract $[22,23]$. Overactivation of the JAK2/STAT3 pathway may have a negative effect on UC. In UC patients, the severity of the intestinal lesion was positively correlated with the expression of STAT3 [22]. To further elucidate the effect of HJD on JAK2/STAT3 pathway, AG490, a specific JAK2 inhibitor, was used to block the JAK2/STAT3 pathway [24]. We found that, similar to that reported about the overexpression of STAT3 and NF-KB caused abnormal activation of JAK2/STAT3 pathway [25], the expressions of JAK2 and STAT3 in the UC group were significantly increased. After administration with HJD, the expressions of JAK2 and STAT3 decreased significantly $(P<0.01)$. This suggested that HJD might inhibit JAK2/STAT3 pathway.

JAK/STAT pathway plays an important role in regulating apoptosis and proliferation. Thus, mono-antibodies of anti-TNF, such as adamumab (HUMIRA ${ }^{\circledR}$ ) and infliximab (REMICADE ${ }^{\circledR}$ ), are used for treatment of inflammatory bowel disease (IBD). Study showed that mono-antibodies inhibited apoptosis of intestinal epithelial cells and promoted mucosal repair [26]. Ki67, a marker of cell proliferation, is abnormally expressed in intestinal inflammation and carcinogenesis, and is associated with intestinal inflammation or proliferation, infiltration, and metastasis of malignant cells [27, 28]. In this paper, we found that Ki67 expression in UC group was significantly higher than control group $(P<0.05)$. However, an increase of Ki67 expression after administration of HJD was observed too. Furthermore, TUNEL staining was used to detect apoptosis of colon epithelial cells. Interestingly, the result showed that the apoptosis in UC group was significantly higher than that in control group $(P<0.01)$, meanwhile the apoptosis was significantly decreased after HJD intervention $(P<0.01)$. These results suggested that HJD could regulate cell proliferation and apoptosis through JAK2/STAT3 pathway [29].
In the development of IBD complicated with intestinal fibrosis, some fibrosis factors, such as transforming growth factor beta (TGF- $\beta$ ), would stimulate the disordered expression of extracellular matrix in the intestinal wall, and then cause intestinal stenosis and fibrosis [30]. This process is closely related to the expression of collagen in the intestinal mucosa. To investigate collagen deposition in the colonic mucosa in UC mice, Masson staining was used to detect collagen expression. The results showed that the collagen deposition in UC group was significantly higher than that in control group, which might be related to the long-term irritation caused by acute inflammation and cell proliferation in the intestinal mucosal. However, the deposition of collagen decreased significantly in HJD group $(P<0.01)$. These results suggest that HJD is beneficial for alleviating intestinal fibrosis. More experiments are necessary to study the mechanisms of HJD on cell proliferation and apoptosis.

\section{Conclusions}

HJD treatment can alleviate intestinal mucosal damage and has the protective effect on UC by downregulating JAK2 and STAT3 expression to reduce inflammation via JAK2/STAT3 pathway.

\section{Abbreviations \\ HJD: Huanglian Jiedu Decoction; HL: Rhizoma coptidis; HB: Cortex phello- dendri chinensis; HQ: Radix scutellariae; ZZ: Fructus gardeniae; UC: Ulcerative colitis; DSS: Dextran sulphate sodium; USAN: Mesalazine; DAl: Disease activity index; CMDI: Colon macroscopic damage index; H\&E: Haematoxylin and eosin; ELISA: Enzyme-linked immunosorbent assay; WB: Western blotting; TUNEL: Terminal deoxynucleotidyl transferase dUTP nick end labelling; 5-ASA: 5-ami- nosalicylic acid; IBD: Inflammatory bowel disease; CMDI: Colon macroscopic damage index; IHC: Immunohistochemistry; RIPA: Radioimmunoprecipitation assay; PVDF: Polyvinylidene difluoride; HRP: Horseradish peroxidase; RT: Room temperature; RH: Relative humidity; IOD: Integrated optical density; AOD: Average optical density; TGF- $\beta$ : Transforming growth factor beta; ANOVA: A one-way analysis of variance; ATT: a-1 antitrypsin.}

\section{Acknowledgements}

We thank LetPub (http://www.LetPub.com) for linguistic assistance during the preparation of this manuscript.

\section{Authors' contributions}

$Z L$ and $J L$ designed the study; $Z L$ and $W X$ wrote the main manuscript text; $\mathrm{ZL}, \mathrm{SX}, \mathrm{YL}$, and $\mathrm{KY}$ performed the animal experiments; GY, QL, and FL analysed the data and prepared the figures. All authors read and approved the final manuscript.

\section{Funding}

This work was supported by National Natural Science Foundation of China (81560674, 81960872), Guangxi Natural Science Foundation (2017GXNSFAA198078), and Guangxi Innovation-Driven Development Project (GUIKEAA17202035, GUIKEAA17202031).

Availability of data and materials

The datasets used in the current study are available from the corresponding author on reasonable request. 


\section{Ethics approval and consent to participate}

The animal care and experimental procedures used in the current study were approved by the Institutional Animal Care and Use Committee of the Guangxi University of Chinese Medicine.

\section{Consent for publication}

Not applicable.

\section{Competing interests}

The authors declare that they have no competing interests.

\section{Author details}

${ }^{1}$ Guangxi University of Chinese Medicine, Nanning 530001, China. ${ }^{2}$ Department of Pharmacy, Guangxi Medical College, Nanning 530023, China. ${ }^{3} \mathrm{Col}-$ lege of Animal Science and Technology, Guangxi University, Nanning 530004, China.

Received: 31 December 2019 Accepted: 29 April 2020 Published online: 08 May 2020

\section{References}

1. Amarapurkar AD, Amarapurkar DN, Rathi P, Sawant P, Patel N, Kamani P, et al. Risk factors for inflammatory bowel disease. A prospective multicenter study. Indian J Gastroenterol. 2018;37:189-95.

2. Ji R, Wang A, Shang H, Chen L, Bao C, Wu L, et al. Herb-partitioned moxibustion upregulated the expression of colonic epithelial tight junctionrelated proteins in Crohn's disease model rats. Chin Med. 2016;11:20.

3. Gao C, Liu L, Zhou Y, Bian Z, Wang S, Wang Y. Novel drug delivery systems of Chinese medicine for the treatment of inflammatory bowel disease. Chin Med. 2019;14:23

4. Becker C, Neurath MF, Wirtz S. The intestinal microbiota in inflammatory bowel disease. ILAR J. 2015;56:192-204.

5. Guo M, Ding S, Zhao C, Gu X, He X, Huang K, et al. Red Ginseng and Semen Coicis can improve the structure of gut microbiota and relieve the symptoms of ulcerative colitis. J Ethnopharmacol. 2015;162:7-13.

6. Li Y, de Haar C, Chen M, Deuring J, Gerrits MM, Smits R, et al. Diseaserelated expression of the IL6/STAT3/SOCS3 signalling pathway in ulcerative colitis and ulcerative colitis-related carcinogenesis. Gut. 2010;59:227-35.

7. Grivennikov S, Karin E, Terzic J, Mucida D, Yu GY, Vallabhapurapu S, et al. IL-6 and Stat3 are required for survival of intestinal epithelial cells and development of colitis-associated cancer. Cancer Cell. 2009:15:103-13.

8. Lee SY, Lee SH, Yang EJ, Kim JK, Kim EK, Jung K, et al. Coenzyme Q10 inhibits Th17 and STAT3 signaling pathways to ameliorate colitis in mice. J Med Food. 2017;20:821-9.

9. Lu J, Wang JS, Kong LY. Anti-inflammatory effects of Huang-Lian-Jie-Du decoction, its two fractions and four typical compounds. J Ethnopharmacol. 2011;134:911-8.

10. Ren W, Zuo R, Wang YN, Wang HJ, Yang J, Xin SK, et al. PharmacokineticPharmacodynamic analysis on inflammation rat model after oral administration of Huang Lian Jie Du Decoction. PLoS ONE. 2016;11:e0156256.

11. Yue R, Zhao $L, H u Y$, Jiang $P$, Wang $S$, Xiang $L$, et al. Metabolomic study of collagen-induced arthritis in rats and the interventional effects of huanglian-jie-du-tang, a traditional chinese medicine. Evid Based Complement Alternat Med. 2013;2013:439690.

12. Zhang Y, Liang Y, He C. Anticancer activities and mechanisms of heatclearing and detoxicating traditional Chinese herbal medicine. Chin Med. 2017;12:20.

13. Mao JW, Tang HY, Wang YD. Influence of rosiglitazone on the expression of PPARgamma, NF-kappaB, and TNF-alpha in rat model of ulcerative colitis. Gastroenterol Res Pract. 2012;2012:845672.
14. Cho CW, Ahn S, Lim TG, Hong HD, Rhee YK, Yang DC, et al. Cynanchum wilfordii polysaccharides suppress dextran sulfate sodium-induced acute colitis in mice and the production of inflammatory mediators from macrophages. Mediators Inflamm. 2017;2017:3859856.

15. Kim Y, Wu AG, Jaja-Chimedza A, Graf BL, Waterman C, Verzi MP, et al. Isothiocyanate-enriched moringa seed extract alleviates ulcerative colitis symptoms in mice. PLoS ONE. 2017;12:e0184709.

16. Xiao H-T, Peng J, Hu D-D, Lin C-Y, Du B, Tsang S-W, et al. Qing-dai powder promotes recovery of colitis by inhibiting inflammatory responses of colonic macrophages in dextran sulfate sodium-treated mice. Chin Med. 2015;10:29.

17. Hussein AH, Freund JN, Reimund JM, Shams A, Yamine M, Leone A, et al. Enteropathogenic e.coli sustains iodoacetamide-induced ulcerative colitis-like colitis in rats: modulation of IL-1 beta, IL-6, TNF-alpha, COX-2, and apoptosisi. J Biol Regul Homeost Agents. 2012;26:515-26.

18. Chen B, She S, Li D, Liu Z, Yang X, Zeng Z, et al. Role of miR-19a targeting TNF-alpha in mediating ulcerative colitis. Scand J Gastroenterol. 2013:48:815-24.

19. Yu XT, Xu YF, Huang YF, Qu C, Xu LQ, Su ZR, et al. Berberrubine attenuates mucosal lesions and inflammation in dextran sodium sulfate-induced colitis in mice. PLoS ONE. 2018;13:e0194069.

20. Hsu YL, Kuo PL, Tzeng TF, Sung SC, Yen MH, Lin LT, et al. Huang-lian-jiedu-tang, a traditional Chinese medicine prescription, induces cell-cycle arrest and apoptosis in human liver cancer cells in vitro and in vivo. J Gastroenterol Hepatol. 2008:23:e290-9.

21. Wu P, Guo Y, Jia F, Wang X. The effects of armillarisin a on serum IL1 beta and IL-4 and in treating ulcerative colitis. Cell Biochem Biophys. $2015 ; 72: 103-6$

22. Lin $H$, Honglang $L$, Weifeng $L$, Junmin $C$, Jiantao $Y$, Junjing $G$. The mechanism of alopolysaccharide protecting ulceralive colitis. Biomed Pharm. 2017:88:145-50.

23. Chu XQ, Wang J, Chen GX, Zhang GQ, Zhang DY, Cai YY. Overexpression of microRNA-495 improves the intestinal mucosal barrier function by targeting STAT3 via inhibition of the JAK/STAT3 signaling pathway in a mouse model of ulcerative colitis. Pathol Res Pract. 2018;214:151-62.

24. Lai IC, Lai G-M, Chow J-M, Lee H-L, Yeh C-F, Li C-H, et al. Active fraction (HS7) from Taiwanofungus camphoratus inhibits AKT-mTOR, ERK and STAT3 pathways and induces CDK inhibitors in CL1-0 human lung cancer cells. Chin Med. 2017;12:33

25. Caprioli F, Stolfi C, Caruso R, Fina D, Sica G, Biancone L, et al. Transcriptional and post-translational regulation of Flip, an inhibitor of Fas-mediated apoptosis, in human gut inflammation. Gut. 2008:57:1674-80.

26. Zeissig S, Bojarski C, Buergel N, Mankertz J, Zeitz M, Fromm M, et al. Downregulation of epithelial apoptosis and barrier repair in active Crohn's disease by tumour necrosis factor alpha antibody treatment. Gut. 2004;53:1295-302.

27. Fratila OC, Ilias TI. COX-2 and Ki-67 immunohistochemical markers in the assessment of long-standing ulcerative colitis associated dysplasia. Rom J Morphol Embryol. 2013:54:143-9.

28. Guan Y, Zhang L, Li X, Zhang X, Liu S, Gao N, et al. Repression of mammalian target of rapamycin complex 1 inhibits intestinal regeneration in acute inflammatory bowel disease models. J Immunol. 2015;195:339-46.

29. Yang SF, Yuan SS, Yeh YT, Wu MT, Su JH, Hung SC, et al. The role of p-STAT3 (ser727) revealed by its association with Ki-67 in cervical intraepithelial neoplasia. Gynecol Oncol. 2005;98:446-52.

30. Liu RM, Desai LP. Reciprocal regulation of TGF-beta and reactive oxygen species. A perverse cycle for fibrosis. Redox Biol. 2015;6:565-77.

\section{Publisher's Note}

Springer Nature remains neutral with regard to jurisdictional claims in published maps and institutional affiliations. 